\title{
NEUROPROTECTIVE ROLE OF BIMOCLOMOL IN ECTOPIC CELL CYCLE IN PARKINSON'S DISEASE: NEW INSIGHTS
}

\begin{abstract}
RENU SHARMA ${ }^{1}$, PRAVIR KUMAR ${ }^{2 *}$
${ }^{1}$ Department of Biotechnology, Molecular Neuroscience and Functional Genomics Laboratory, Delhi Technological University (Formerly DCE), New Delhi, India. ${ }^{2}$ Department of Biotechnology, Molecular Neuroscience and Functional Genomics Laboratory, Delhi Technological University (Formerly DCE), New Delhi, India. Email: pravirkumar@dce.edu/kpravir@gmail.com
\end{abstract}

Received: 17 February 2017, Revised and Accepted: 11 March 2017

\section{ABSTRACT}

Objective: Parkinson's disease (PD) is a debilitating age-related neurodegenerative disease characterized by the canonical formation of intracellular Lewy bodies comprising $\alpha$-synuclein protein. Despite the knowledge of factors causing PD, it remains irreversible and incurable. Recent studies have highlighted the physiological and pathological involvement of cell cycle proteins in PD. The intriguing relationship between PARK2 and cyclin E which leads to upregulation of cyclin $\mathrm{E}$ in the absence of functional PARK2 contributes heavily in the onset and progression of PD. The objective of this study is to explore neuroprotective action of bimoclomol in attenuating the level of cyclin $\mathrm{E}$ and inhibiting post-mitotic cell division led neurodegeneration in PD.

Methods: We employed various in silico methods such as drug-likeness parameters, namely, Lipinski filter analysis, Ghose parameters, Veber rules, absorption, distribution, metabolism, and excretion - toxicity analysis, pharmacophore based target prediction, active site prediction, and molecular docking studies.

Results: The binding of bimoclomol inhibited cyclin E, thereby, attenuating post-mitotic cell division led neurodegeneration in PD.

Conclusion: This study outlines the novel potential of bimoclomol in attenuating cyclin E led neuronal death in PD which may be mediated by heat shock proteins (HSP70).

Keywords: Parkinson's disease, Bimoclomol, Cell cycle, Heat shock proteins 70, Therapeutics.

(c) 2017 The Authors. Published by Innovare Academic Sciences Pvt Ltd. This is an open access article under the CC BY license (http://creativecommons. org/licenses/by/4. 0/) DOI: http://dx.doi.org/10.22159/ajpcr.2017.v10i6.17971

\section{INTRODUCTION}

Parkinson's disease (PD) is the second most common age-related neurodegenerative disorder affecting $2 \%$ of population aged above 65 years in industrialized nations. It is characterized by pathogenic protein burden and intracellular inclusion body formation, namely, Lewy bodies and Lewy neurites constituted by $\alpha$-synuclein protein. The clinical cardinal features of PD include resting tremor, bradykinesia, postural instability and rigor, often accompanied by impaired cognition [1]. These symptoms are an outcome of dopaminergic neuronal loss in the substantia nigra pars compacta region of the brain.

Genetic and animal studies have outlined various causative phenomenon in PD including mutations in genes predominantly; Parkin, $\alpha$-synuclein, PINK1 and DJ-1, oxidative stress, aging, impaired ubiquitin proteasome system, and dysfunctional mitochondrial system. Despite the availability of this knowledge, the etiology of PD remains incurable and irreversible. Moreover, recent studies have highlighted the involvement of aberrant cell cycle in PD [2]. Interestingly; cell cycle proteins share a very intimate relationship with proteins of PD, physiologically as well as pathologically. The PD-associated gene; PINK1 was shown to promote cell cycle, and PINK1 deletion reversed cell proliferation [3]. Further, the ATM gene responsible for DNA damage response and apoptosis has been found to be activated along with retinoblastoma protein leading to neuronal death in $\mathrm{MPP}^{+}$induced PD model [4]. However, the most crucial and strong correlation between cell cycle and PD is provided by the association of cyclin $E$ and PARK2. Cyclin E is $G_{1} / S$ phase marker of the cell cycle and also a substrate of ubiquitin E3 ligase PARK2. Mutations associated with loss of functional PARK2 are linked with cyclin E enrichment led cell cycle and apoptosis through p53 and Bax in PD. Further, mutated PARK2/cyclin E events evoked upregulation of Wnt/ $\beta$ catenin and EGFR/AKT signal transduction pathways [5]. This intriguing PARK2/cyclin E relation led to speculations that compounds which can bind to and attenuate the level of cyclin E can ameliorate post-mitotic cell division led neurodegeneration in PD.

Numerous studies have highlighted the neuroprotective action of heat shock proteins (HSP) particularly, HSP70. Further, HSP70 is closely associated with cell cycle regulation and was also found to interact with cyclin E in inclusion body myositis and polymyositis [6]. Therefore, we carried out comprehensive data mining for HSP70 inducers in neurodegenerative diseases and selected bimoclomol among 20 compounds based on drug-likeness, pharmacokinetics, and blood-brain barrier (BBB) permeability (unpublished results). Bimoclomol is a hydroxylamine derivative with molecular formula $\mathrm{C}_{14} \mathrm{H}_{20} \mathrm{ClN}_{3} \mathrm{O}_{2}$ and molecular weight of $297.783 \mathrm{~g} / \mathrm{mol}$. It has been shown to elicit protective effects through induction of HSP27, HSP70, and HSP90. Moreover, bimoclomol is currently under Phase II trials in amyotrophic lateral sclerosis disease [7]. Therefore, we investigated the neuroprotective action of bimoclomol through attenuation of cyclin $\mathrm{E}$ in PD. Various virtual screening methods such as Lipinski filter, Ghose and Veber parameters, pharmacophore modeling based target prediction, and ADME analysis were employed to check the efficacy of bimoclomol as a neuroprotective agent. Further, we studied the cyclin E inhibiting potential of bimoclomol through molecular docking studies.

\section{METHODS}

Retrieval of ligand-protein structure

The SDF file of bimoclomol was retrieved from the PubChem database (http://www.pubchem.ncbi.nlm.nih.gov/). The pubChem database stores physiochemical and biological information of compounds from 
three different databases. The protein data bank (PDB) file of cyclin E was retrieved from PDB (http://www.rcsb.org/pdb/home/home.do).

\section{Drug-likeliness analysis}

The drug-likeness of bimoclomol was tested through Lipinski filter analysis which is used to test compounds for drug ability. The Lipinski's rule of five is: (a) Molecular mass $<500$ Dalton, (b) lipophilicity $(\log \mathrm{P})<5$, (c) hydrogen bond donors <5, (d) hydrogen bond acceptors $<10$, and (e) molar refractivity between 40 and 130 [8]. In addition, two other parameters; Ghose filter and Veber rules were employed for drug-likeness screening (www.swissadme.ch/index.php). The qualifying parameters of Ghose filter are (a) molecular weight 160-480, (b) number of atoms 20-70, (c) molar refractivity 40-130, (d) molar refractivity - 0.4-5.6, and (e) polar surface area $<140$ [9]. The Veber rules are (a) rotatable bond count $\leq 10$ and (b) polar surface area $\leq 140$ [10].

Absorption, distribution, metabolism, and excretion - toxicity (ADMET) analysis

The toxicity profiling of bimoclomol was carried out through the online tool Swiss ADME (www.swissadme.ch/index.php). The Swiss ADME tool assessed the ligand on various parameters such as logP, hydrophilic nature $(\log S)$, and BBB permeability.

\section{Pharmacophore based target prediction}

A pharmacophore is a spatial arrangement of steric and electronic properties of a compound responsible for its biological response against a particular target. Pharmacophore-based target prediction of bimoclomol was done with web server PharmMapper (http://59.78.96.61/pharmmapper/index.php) [11].

\section{Active site prediction}

The active sites of cyclin E were predicted using the pockdrug tool (http://pockdrug.rpbs.univ-paris-diderot.fr/cgi-bin/index. py?page=home) [12]. The PDB structure of cyclin E was uploaded, and active sites were predicted using fpocket estimation and setting ligand proximity threshold at 5.5 .

\section{Preparation of protein and ligand for docking}

Cyclin E and bimoclomol were prepared for docking using the online docking server (http://www.dockingserver.com/web) [13]. The protein was cleaned, and chain A of cyclin E was selected for docking. The protein and ligand charge was calculated using Gasteiger method, and default solvation parameters were set. The ligand geometry was optimized using the MMFF94 method. Further, all non-polar $\mathrm{H}_{2}$ atoms were merged, rotatable bonds defined and $\mathrm{pH}$ set to 7.0 .

\section{Molecular docking}

The optimized proteins and ligands were used for molecular docking studies using the online docking server (http://www.dockingserver. com/web). The Autodock tool was used for adding Kollman united atom type charges, essential $\mathrm{H}_{2}$ atoms, and solvation parameters. Affinity grid maps were generated with $0.375 \AA$ spacing [14]. Further, the van der Waals and electrostatic interactions were calculated using Autodock parameter set and distance-dependent dielectric functions, respectively. Furthermore, the Lamarckian genetic algorithm and Soils and Wets local search method was used for docking simulations [15]. During docking, all rotatable torsions were dropped. Every docking study was arrived after 10 different runs with a cut off energy estimation of 250000 . Finally, translational step with $0.2 \AA$, torsion and quaternion steps of five were used with a population size of 150 .

\section{RESULTS}

\section{Protein-ligand structure}

The 3D structures of bimoclomol and cyclin E were retrieved from docking server and PDB, respectively (Fig. 1).

\section{Screening for drug-likeness and ADMET analysis of compounds} Bimoclomol passed all the parameters related to drug-likeness screening, namely, Lipinski, Ghose and Veber. Most importantly, it can cross the BBB and has high pharmacokinetics values (Table 1).
Pharmacophore based target prediction

The pharmacophore based target prediction of bimoclomol revealed mitogen-activated protein kinase 14 as one of the top 10 targets with a fit score of 3.521 and Z-score value of -0.408482 which supported our premise of its strong potential in inhibiting cell cycle (Fig. 2).

\section{Active site prediction}

Out of top 10 pockets, cyclin E had best pocket at $\mathrm{P} 20$ with a drug ability score of 0.94 and 0.01 standard deviation (Fig. 3). The volume of given pocket was 551.26 cubic angstroms and 14 residues were involved in interaction at this site.

\section{Molecular docking of bimoclomol with cyclin E}

Bimoclomol bound to cyclin E at P20 pocket and same residues as predicted were involved in the interaction (Fig. 4). The estimated free energy of binding for cyclin $\mathrm{E}$ and bimoclomol was $-5.07 \mathrm{kcal} / \mathrm{mol}$, and total intermolecular energy was $-6.48 \mathrm{kcal} / \mathrm{mol}$ (Table 2). There were two $\mathrm{H}_{2}$ bond formations involving GLU188 and LYS186 with bond energies of $-0.2603 \mathrm{kcal} / \mathrm{mol}$ and $-0.2271 \mathrm{kcal} / \mathrm{mol}$, respectively. Further, a hydrophobic bond was formed with HIS147 $(-0.6293 \mathrm{kcal} / \mathrm{mol})$

\section{DISCUSSION}

$\mathrm{PD}$ is the second most common age-related neurodegenerative disease affecting those aged above 60 years. Despite the knowledge of several factors which contribute in the occurrence and progression of PD, the exact cause and cure remain elusive. Ectopic activation of the cell cycle in terminally differentiated neurons is a recently known phenomenon which has been shown to drive neurodegeneration through actual DNA

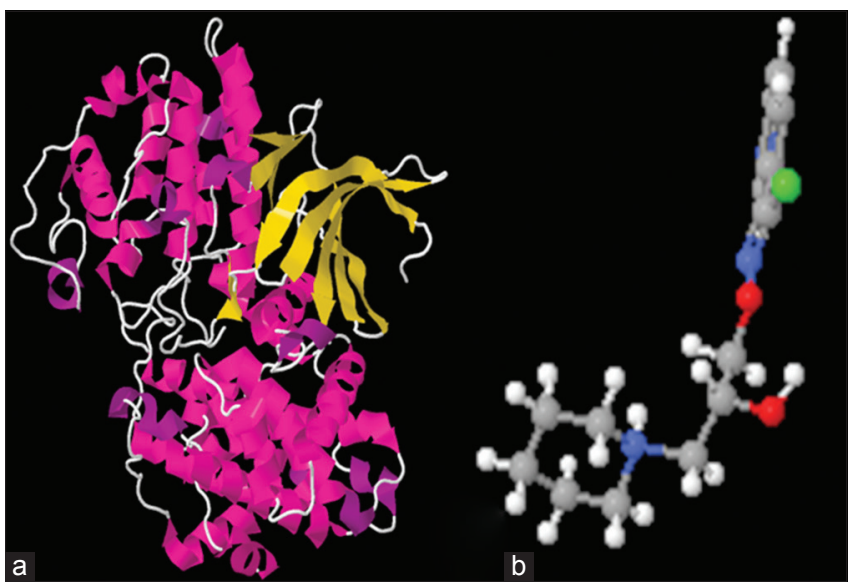

Fig. 1: Three-dimensional structure of cyclin E (a) and bimoclomol (b)

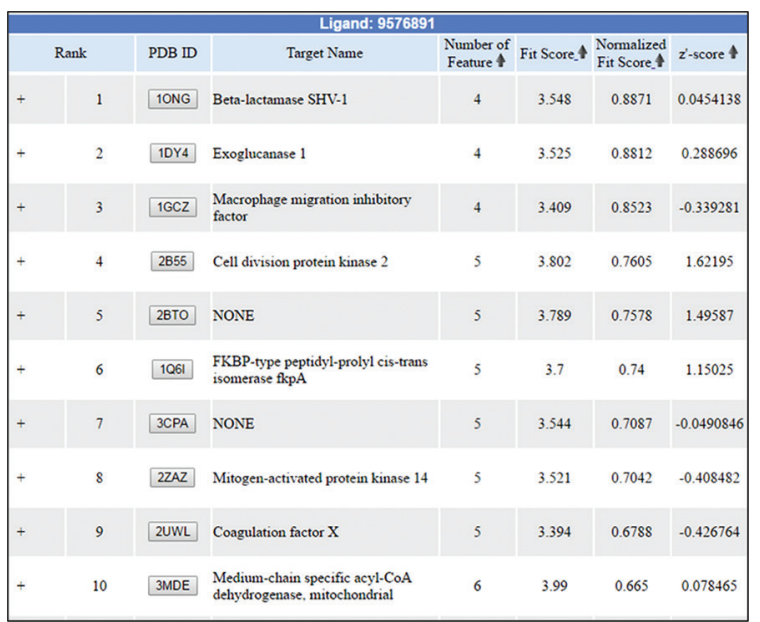

Fig. 2: Pharmacophore based target prediction of bimoclomol 


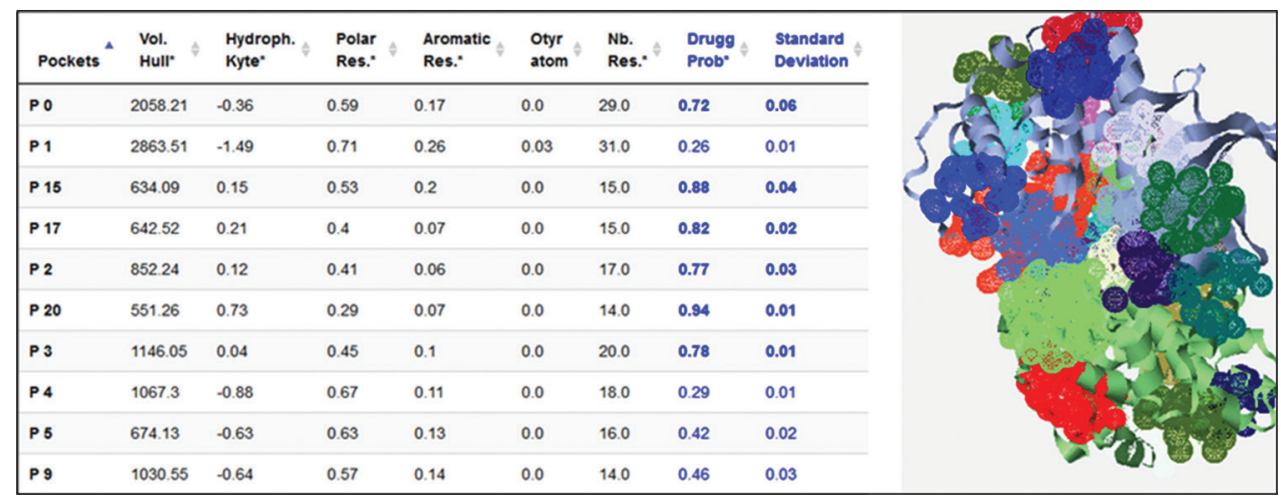

Fig. 3: Active sites of cyclin E

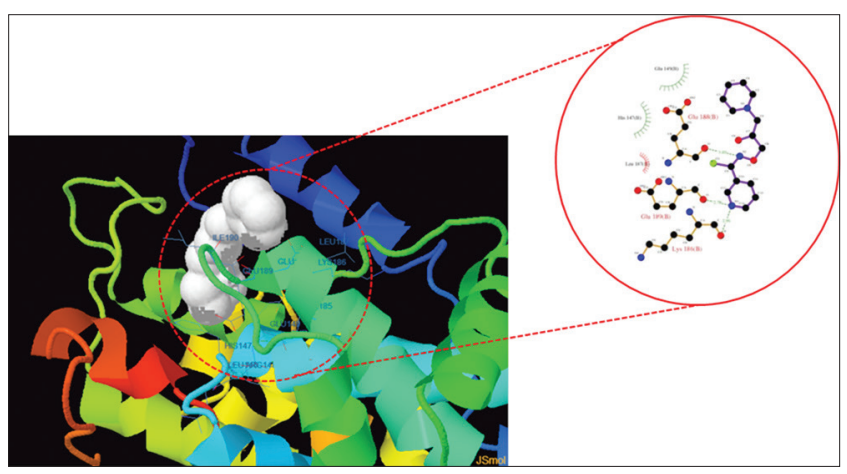

Fig. 4: Docking of bimoclomol with cyclin $\mathrm{E}$ and residues involved (inset)

Table 1: ADMET analysis of Bimoclomol

\begin{tabular}{ll}
\hline GI permeability & High \\
\hline LogS (ESOL) & -2.9 \\
XLogP3 & 2.21 \\
Bioavailability score & 0.55
\end{tabular}

ADMET: Absorption, distribution, metabolism, and excretion - toxicity,

GI: Gastrointestinal, ESOL: Estimated aqueous solubility

Table 2: Energies of binding for cyclin E and bimoclomol

\begin{tabular}{ll}
\hline Estimated free energy of binding & $-5.07 \mathrm{Kcal} / \mathrm{mol}$ \\
Estimated inhibition constant & $191.27 \mathrm{uM}$ \\
vdW+Hbond+desolv energy & $-4.55 \mathrm{Kcal} / \mathrm{mol}$ \\
Electrostatic energy & $-1.93 \mathrm{Kcal} / \mathrm{mol}$ \\
Total intermolecular energy & $-6.48 \mathrm{Kcal} / \mathrm{mol}$ \\
Interacting surface & 541.668 \\
\hline
\end{tabular}

synthesis followed by apoptosis [2]. Moreover, PD-associated proteins have shared a very intimate relation with cell cycle markers. The G1/S phase marker cyclin E is a substrate for PARK2 and participates in ubiquitination process. However, mutations in PARK2 led to the loss of function thereby, resulting in cyclin E accumulation which in turn, activated E2F1 and triggered neuronal death in PD [5]. Thus, it seems imperative to design therapeutic strategies aimed at attenuating the level of cyclin E to inhibit the cascade of neuronal death in PD.

Various biomolecules such as curcumin elicited HSP70 activity and provided protection against neuronal dysfunction, various cancers, and in vascular diseases [16-18]. Bimoclomol is a hydroxylamine derivative which is nontoxic and elicited its protective effect through HSP induction; including HSP70 [19]. HSPs are molecular chaperones which are upregulated in the cell to protect it against heat, ROS and hypoxia. HSP70 has been shown to promote neuronal survival by mediating the activation of pro-survival signaling cascades and through autophagy induction [2]. Interestingly, HSP70 has been shown to interact with cyclin $\mathrm{E}$ in $\mathrm{A} \beta$ induced cell cycle re-entry in inclusion body myositis and polymyositis [6]. Taken together, all these data provide convincing evidence of using HSP70 inducing compound such as bimoclomol in attenuating the level of cyclin $\mathrm{E}$ and in turn, inhibit the cascade of neuronal death in PD.

In this study, we tested the drug-able efficacy of bimoclomol for targeting cyclin E in PD. Emphasis was laid on pharmacokinetic analysis as aqueous solubility, and dissolution in gastrointestinal fluids are defining parameters of in vivo bioavailability of an orally administered drug [20]. Similarly, the lipophilicity of a drug directs physiological properties such as rate of metabolism, transport across cell membrane and interaction with binding sites of the receptor. Further, CNS drugs should have $\log \mathrm{P}<4[21,22]$. The $\log \mathrm{P}$ value for bimoclomol was found to be 2.21 .

However, the most important property required of a compound intended to be a neuroprotective agent is BBB permeability. Bimoclomol qualified all the above-mentioned parameters and scored well on pharmacokinetics, bioavailability score and could cross the BBB. Finally, molecular docking studies indicated that bimoclomol can bind to and attenuate the level of cyclin $\mathrm{E}$ and possibly, halt or inhibit cell cycle re-entry mediated neuronal death in PD. These findings can be validated through in vitro and in vivo cell cycle studies in PD.

\section{CONCLUSION}

The results of our study provide the novel potential of bimoclomol in attenuating the level of cyclin $\mathrm{E}$ which has wider implications in inhibiting cell cycle re-entry mediated neurodegeneration in PD.

\section{ACKNOWLEDGMENT}

The authors would like to thank senior management of Delhi Technological University for their encouragement and support. The authors would also like to thank University Grants Commission for providing Senior Research Fellowship to R.S.

\section{REFERENCES}

1. Ebrahimi-Fakhari D, Wahlster L, McLean PJ. Molecular chaperones in Parkinson's disease - Present and future. J Parkinsons Dis 2011;1(4):299-320.

2. Sharma R, Kumar D, Jha NK, Jha SK, Ambasta RK, Kumar P. Reexpression of cell cycle markers in aged neurons and muscles: Whether cells should divide or die? Biochim Biophys Acta 2017;1863(1):324-36.

3. O'Flanagan CH, Morais VA, Wurst W, De Strooper B, O'Neill C. The Parkinson's gene PINK1 regulates cell cycle progression and promotes cancer-associated phenotypes. Oncogene 2015;34(11):1363-74.

4. Camins A, Pizarro JG, Alvira D, Gutierrez-Cuesta J, de la Torre AV, Folch $\mathrm{J}$, et al. Activation of ataxia telangiectasia muted under experimental models and human Parkinson's disease. Cell Mol Life Sci 
2010;67(22):3865-82

5. Feng DD, Cai W, Chen X. The associations between Parkinson's disease and cancer: The plot thickens. Transl Neurodegener 2015;4:20.

6. Kwon B, Kumar P, Lee HK, Zeng L, Walsh K, Fu Q, et al. Aberrant cell cycle reentry in human and experimental inclusion body myositis and polymyositis. Hum Mol Genet 2014;23(14):3681-94

7. Keppel Hesselink JM. Bimoclomol and arimoclomol: HSP-co-inducers for the treatment of protein misfolding disorders, neuropathy and neuropathic pain. J Pain Relief 2016;6:279.

8. Lipinski CA, Lombardo F, Dominy BW, Feeney PJ. Experimental and computational approaches to estimate solubility and permeability in drug discovery and development settings. Adv Drug Deliv Rev 2001;46(1-3):3-26

9. Ghose AK, Viswanadhan VN, Wendoloski JJ. A knowledge-based approach in designing combinatorial or medicinal chemistry libraries for drug discovery 1 . A qualitative and quantitative characterization of known drug databases. J Comb Chem 1999;1(1):55-68.

10. Veber DF, Johnson SR, Cheng HY, Smith BR, Ward KW, Kopple KD. Molecular properties that influence the oral bioavailability of drug candidates. J Med Chem 2002;45(12):2615-23.

11. Liu X, Ouyang S, Yu B, Liu Y, Huang K, Gong J, et al. PharmMapper server: A web server for potential drug target identification using pharmacophore mapping approach. Nucleic Acids Res 2010;38:W609-14

12. Hussein HA, Borrel A, Geneix C, Petitjean M, Regad L, Camproux AC. PockDrug-server: A new web server for predicting pocket druggability on holo and apo proteins. Nucleic Acids Res 2015;43:W436-42.

13. Bikadi Z, Hazai E. Application of the PM6 semi-empirical method to modeling proteins enhances docking accuracy of AutoDock.
J Cheminform 2009;1:15.

14. Morris GM, Goodsell DS. Automated docking using a genetic lamarckian algorithm and an empirical binding free energy function. J Comput Chem 1998;19(14):1639-62.

15. Soils FJ, Wets RJ. Minimization by random search techniques. Math Oper Res 1981;6(1):19-30.

16. Xia C, Cai Y, Li S, Yang J, Xiao G. Curcumin increases HSP70 expression in primary rat cortical neuronal apoptosis induced by gp 120 V3 loop peptide. Neurochem Res 2015;40:1996-2005.

17. Sharma S, Kumar V. In vitro cytotoxicity effect on mcf-7 cell line of co-encapsulated artesunate and curcumin liposome. Int J Pharm Pharm Sci 2017;9(3):123-8

18. Joshi H, Bhandari U, Panda BP. To assess the potential of curcumin against gut microbiota-induced alteration in choline metabolism in c57bl/6j mice. Int J Pharm Pharm Sci 2017;9(3):215-26.

19. Deane CA, Brown IR. Induction of heat shock proteins in differentiated human neuronal cells following co-application of celastrol and arimoclomol. Cell Stress Chaperones 2016;21(5):837-48.

20. Khadka P, Jieun R, Kim H, Kim I, Kim JT, Kim H, et al. Pharmaceutical particle technologies: An approach to improve drug solubility, dissolution and bioavailability. Asian J Pharm Sci 2014;9(6):304-16.

21. Chico LK, Van Eldik LJ, Watterson DM. Targeting protein kinases in central nervous system disorders. Nat Rev Drug Discov 2009;8(11):892-909.

22. Wager TT, Hou X, Verhoest PR, Villalobos A. Moving beyond rules: The development of a central nervous system multiparameter optimization (CNS MPO) approach to enable alignment of druglike properties. ACS Chem Neurosci 2010;1(6):435-49. 